\title{
High-temperature characterisation and analysis of leakage- current-compensated, low-power bandgap temperature sensors
}

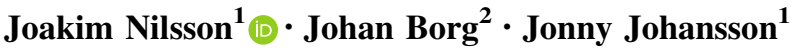

Received: 19 December 2016/Revised: 25 April 2017/Accepted: 15 June 2017/Published online: 28 June 2017

(c) The Author(s) 2017. This article is an open access publication

\begin{abstract}
This paper analyses leakage current compensation techniques for low-power, bandgap temperature sensors. Experiments are conducted for circuits that compensate for collector-substrate, collector-base, bodydrain and source-body leakage currents in a Brokaw bandgap sensor. The sensors are characterised and their failure modes are analysed at temperatures from 60 to $230^{\circ} \mathrm{C}$. It is found that the most appropriate compensation circuit depends on the accuracy requirements of the application and on whether a stable reference voltage is required by other parts of the circuit. Experiments show that the power consumption is dominated by leakage current at high temperatures. One type of sensor was seen to consume $260 \mathrm{nW}$ at $60^{\circ} \mathrm{C}, 2.1 \mu \mathrm{W}$ at $200^{\circ} \mathrm{C}$ and $14 \mu \mathrm{W}$ at $230^{\circ} \mathrm{C}$. This work is motivated by the need to accurately monitor the temperature of power semiconductors in order to predict emerging faults in power semiconductor modules, a task for which cheap, single-chip, low-power, hightemperature, wireless bandgap temperature sensors are appropriate.
\end{abstract}

Keywords Bandgap · Condition monitoring · High temperature - Leakage current - Low power - Power semiconductor - Temperature sensor - Voltage reference

Joakim Nilsson

joanil@1tu.se

Johan Borg

j.borg@imperial.ac.uk

Jonny Johansson

jonny@1tu.se

1 Department of Computer Science, Electrical and Space Engineering, Luleå University of Technology, Luleå, Sweden

2 Department of Physics, Imperial College, London, UK

\section{Introduction}

High-temperature sensors are used in power electronic equipment to monitor the temperature of power semiconductors [17], and their use in this area is the main motivation behind this work. The ageing of the solder interface between power semiconductor and cooling baseplate causes an increase in operating temperature of the device over time and can eventually result in the device's destruction [4]. If a temperature rise can be detected before a device fault occurs, preventive maintenance can be performed, and catastrophic failure can be avoided.

Systems that measure the temperature of power semiconductors by using direct-contact sensors are not known by the authors to exist, but would provide advantages in terms of accuracy and portability between modules [7]. This work aims to enable the manufacturing of such a system by demonstrating a low-power integrated circuit (IC) temperature sensor that is able to operate up to at least $230^{\circ} \mathrm{C}$.

Low-power, high-temperature sensors can be used in conjunction with on-chip coils for power transfer to monitor the temperature of power semiconductors [8]. The concept is illustrated in Fig. 1. Here, the temperature sensors are glued in direct contact with the power semiconductors to provide accurate measurements while being galvanically isolated from the main measurement system, as communication and power supply are implemented wirelessly through radio-frequency identification (RFID) technology.

One challenge in designing low-power, high-temperature bandgap temperature sensors is the exponential increase in leakage current with increasing temperature [7]. These leakage currents arise in reverse-biased p-n junctions [15]. Unless compensated for, this effect will set the upper operating temperature limit of a sensor. 


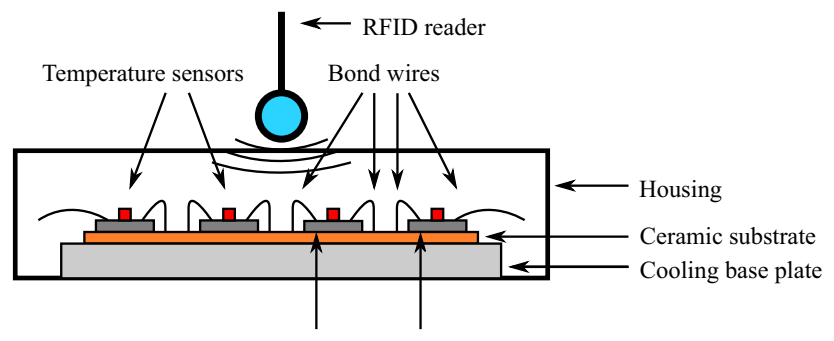

Power semiconductors

Fig. 1 Schematic view of the cross-section of a wire-bond power semiconductor module whose devices are being monitored by singlechip temperature sensors powered by a nearby RFID reader [8]

This paper presents high-temperature characterisations of low-power temperature sensors by providing and analysing measured data of manufactured ICs in a $0.18 \mu \mathrm{m}$ psubstrate CMOS process with vertical bipolar junction transistors (BJTs). The sensors are Brokaw bandgap temperature sensors with collector-substrate (CS) leakage current compensation from [11] for bipolar transistors. We also include circuitry for collector-base (CB) leakage current compensation as well as for body-drain (BD) and source-body (SB) leakage current compensation for metaloxide semiconductor field effect transistors (MOSFETs). In these circuits, the NMOS and PMOS bodies are p-wells and n-wells connected to ground and supply voltage, respectively. Experiments are performed for temperatures up to $230^{\circ} \mathrm{C}$ to characterise circuits with no leakage current compensation circuitry, with the compensation circuit found in [11] (previously characterised up to $175^{\circ} \mathrm{C}$ ) and with said compensation circuit plus the compensation circuit demonstrated in [7], which has previously only been characterised through simulation up to $175^{\circ} \mathrm{C}$.

The experiments show that the use of $\mathrm{CB} / \mathrm{BD} / \mathrm{SB}$ leakage current compensation circuits enable operation up to at least $230^{\circ} \mathrm{C}$. They further show that many of the sensors that do not use this type of compensation circuit fail at temperatures around 200 to $210^{\circ} \mathrm{C}$.

In Sect. 2, we present a survey of previous work on leakage current compensation techniques for bandgap circuits as well as the theory behind the sensor presented in this work. In Sect. 3, the experimental set-up is described, with the experimental results presented in Sect. 4. Section 5 presents a discussion of the results and compares them to the results of other works. Conclusions are presented in Sect. 6.

\section{Low-power bandgap temperature sensors}

The Brokaw bandgap reference [3] is commonly used to measure temperatures. One implementation is depicted in Fig. 2. The basic idea is that the base-emitter (BE) voltage,
$V_{\mathrm{BE}}$, of a BJT has a negative temperature coefficient, whereas the difference in voltage between a larger and smaller BJT, $\Delta V_{\mathrm{BE}}$, has a positive temperature coefficient and is thus proportional to absolute temperature (PTAT). $V_{\mathrm{BE}}$ is given in [5] as

$V_{\mathrm{BE}}=V_{\mathrm{G} 0}-c_{\mathrm{T}} T+V N L(T)$.

Here, $V_{\mathrm{G} 0} \approx 1.2 \mathrm{~V}$ is the extrapolated bandgap voltage at $0 \mathrm{~K}, c_{\mathrm{T}}$ is a constant temperature coefficient, $T$ is the temperature, and $V N L(T)$ represents temperature-dependent, non-linear terms. In this work, we ignore $V N L(T)$ because it is insignificant considering our desired accuracy and substantially smaller than the errors introduced due to leakage at high temperatures.

By summing and scaling $\Delta V_{\mathrm{BE}}$ and $V_{\mathrm{BE}}$, two new voltages are generated; one voltage that is constant over temperature, VREF, and one that is PTAT, VPTAT. The ratio of these voltages, $V P T A T / V R E F$, can be used in conjunction with an analog-to-digital converter (ADC) to obtain temperature measurements.

\subsection{Operation of the Brokaw bandgap reference}

Ignoring the effects of $Q_{3}$ for now, the circuit of Fig. 2 operates by having the current mirror $M_{1}-M_{2}$ force equal collector currents $I_{\mathrm{C} 1}$ and $I_{\mathrm{C} 2}$ through $Q_{1}$ and $Q_{2}$ using the common source transistor $M_{3}$ to feedback an error signal into the bases of $Q_{1}$ and $Q_{2}$.

Because $Q_{1}$ and $Q_{2}$ differ in size and carry equal collector currents, they are biased at different current densities, and thus, a voltage $\Delta V_{\mathrm{BE}}$ develops across their emitters, that is, over $R_{1}$. Because $\Delta V_{\mathrm{BE}}$ is PTAT, the current through $R_{1}, I_{\mathrm{E} 2}$, will be PTAT. Assuming equal emitter currents, $I_{\mathrm{E} 1}=I_{\mathrm{E} 2}=I_{\mathrm{E}}$, the current through $R_{2}$ $\left(2 I_{\mathrm{E}}\right)$ will also be PTAT, and therefore VPTAT will be

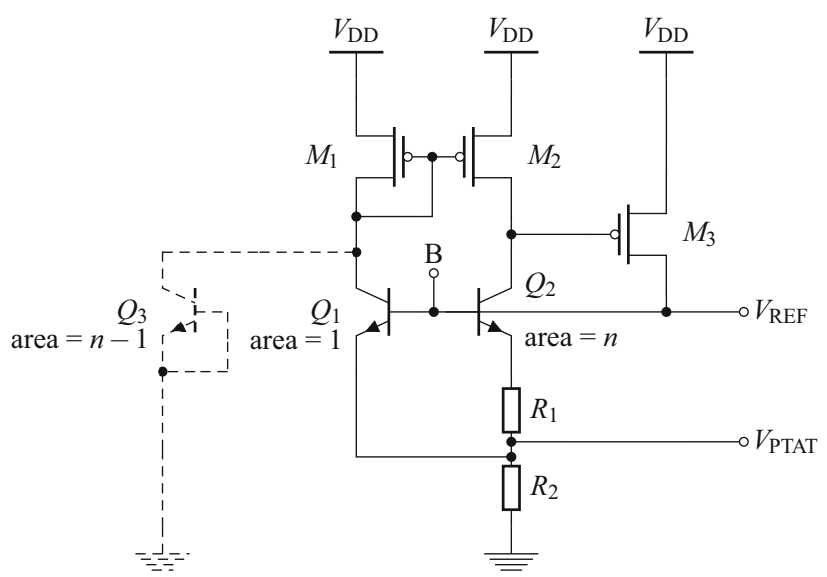

Fig. 2 Brokaw bandgap reference circuit as implemented in [11]. For simplicity, the start-up circuit is shown sepearately in Fig. 3 and is connected to this circuit through their common node B 
PTAT. The voltage $V R E F$ will be the sum of VPTAT and the base-emitter voltage of $Q_{1}, V_{\mathrm{BE} 1}$. Thus, scaling the ratio of $R_{2} / R_{1}$ appropriately makes $V R E F$ independent of temperature and equal to $V_{\mathrm{G} 0}$ because the first-order temperature-dependent terms are cancelled.

An expression for VPTAT is given in [3] as

$V P T A T=2 \frac{R_{2}}{R_{1}} \frac{k T}{q} \ln \frac{J_{\mathrm{C} 1}}{J_{\mathrm{C} 2}}$,

where $k$ is Boltzmann's constant, $T$ is the temperature and $J_{\mathrm{C} 1}$ and $J_{\mathrm{C} 2}$ are the respective collector current densities of $Q_{1}$ and $Q_{2}$. If $I_{\mathrm{C} 1}=I_{\mathrm{C} 2}$, we can write

$V P T A T=2 \frac{R_{2}}{R_{1}} \frac{k T}{q} \ln \left(n \frac{I_{\mathrm{C} 1}}{I_{\mathrm{C} 2}}\right)$,

where $n$ is the area ratio between transistors $Q_{2}$ and $Q_{1}$.

To avoid the operating point at which no current flows through $Q_{1}$ and $Q_{2}$, a start-up circuit must be introduced. This circuit is shown in Fig. 3 and is connected to the circuit of Fig. 2 through their common node B. It operates by having the pull-up network $M_{\mathrm{S} 3}-M_{\mathrm{S} 4}$ turn $M_{\mathrm{S} 2}$ on unless there is a voltage $V R E F$ at node $\mathrm{B}$ to turn $M_{\mathrm{S} 1}$ on, which then in turn drives $M_{\mathrm{S} 2}$ off. This arrangement enforces a non-zero voltage, $V R E F$ at node B.

\subsection{Effects of collector-substrate leakage current in bandgap circuits}

Leakage current poses a challenge in high-temperature IC design [15]. Figure 4 shows an extended version of the schematic in Fig. 2, where major sources of leakage current due to reverse-biased p-n junctions are shown and indicated as current sources. In this figure, CS leakage occurs in $Q_{1}, Q_{2}$ and $Q_{3}$. Because leakage current in reverse-biased p-n junctions increases exponentially with increasing temperature [14], at high temperatures, the leakage currents of a low-power bandgap temperature sensor become significant as their magnitudes become comparable to that of the sensor's quiescent current. One aspect of how leakage current adversely affects bandgap circuits has been studied by Radoiaş et al. [10, 11]. They found that, at high

Fig. 3 Start-up circuit for the Brokaw bandgap circuit of Fig. 2. This circuit is connected to the bandgap circuit through their common node B

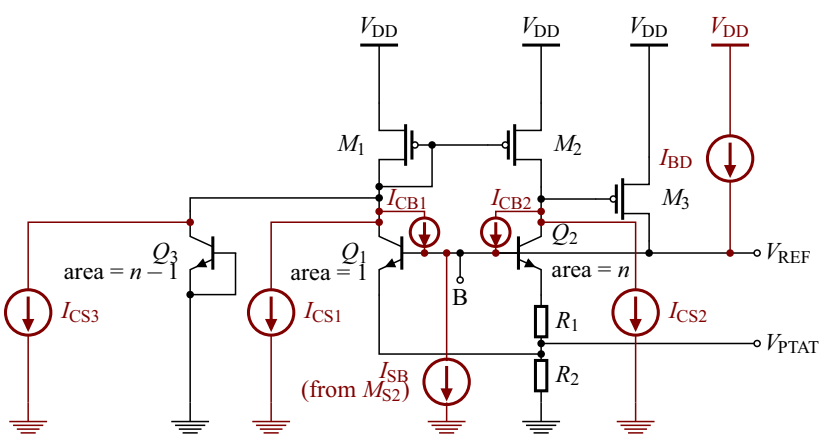

Fig. 4 Brokaw bandgap circuit with leakage current caused by reverse-biased $p-n$ junctions represented as current sources. The subscripts $\mathrm{CS}, \mathrm{CB}, \mathrm{BD}$ and $\mathrm{SB}$ indicate $\mathrm{CS}, \mathrm{CB}, \mathrm{BD}$ and $\mathrm{SB}$ leakage current, respectively

temperatures, CS leakage in the main bipolar pair causes the collector currents $I_{\mathrm{C} 1}$ and $I_{\mathrm{C} 2}$ to become mismatched due to differing sizes of the respective transistors.

The source-drain currents of $M_{1}$ and $M_{2}$ are the sum of their respective associated collector currents, $I_{\mathrm{C} 1}$ and $I_{\mathrm{C} 2}$, and leakage currents, $I_{\mathrm{CS} 1}$ and $I_{\mathrm{CS} 2}$, being drawn from the collectors of $Q_{1}$ and $Q_{2}$. Because $Q_{2}$ is larger than $Q_{1}$, $I_{\mathrm{CS} 2}>I_{\mathrm{CS} 1}$. The current mirror $M_{1}-M_{2}$ and the feedback loop through $M_{3}$ will keep $I_{\mathrm{C} 1}+I_{\mathrm{CS} 1}=I_{\mathrm{C} 2}+I_{\mathrm{CS} 2}$, by increasing the drain current of $M_{3}, I_{\mathrm{D} 3}$, supplied to the bases of $Q_{1}$ and $Q_{2}$. As the BE junction of $Q_{1}$ has a lower impedance than that of $Q_{2}, I_{\mathrm{C} 1}$ will increase more than $I_{\mathrm{C} 2}$. Thus, taking leakage currents into account, the ratio $I_{\mathrm{C} 1} / I_{\mathrm{C} 2}$ increases.

It can be seen from Eq. (3) that VPTAT will increase from an increase in collector current ratio, $I_{\mathrm{C} 1} / I_{\mathrm{C} 2}$. This increase will also be seen in VREF because $V R E F$ is the sum of VPTAT and $V_{\mathrm{BE} 1}$. Thus, at high temperatures, VPTAT/VREF increases beyond the level predicted if leakage current is not considered.

When the temperature, and hence CS leakage currents, become large enough, the collector voltages of $Q_{1}$ and $Q_{2}$ decrease and eventually the CB junctions of $Q_{1}$ and $Q_{2}$ become forward-biased. This causes a part of $I_{\mathrm{D} 3}$ to go through the CB junctions of $Q_{1}$ and $Q_{2}$, reducing the current through their $\mathrm{BE}$ junctions. Thus, $I_{\mathrm{C} 1}$ and $I_{\mathrm{C} 2}$ are reduced, effectively reducing $V P T A T$ and $V R E F$. At sufficiently high temperatures, this effect prevents the circuit from operating as a bandgap reference, thus disabling its ability to function as a temperature sensor.

Radoiaş et al. compensated for unequal CS leakage by introducing another transistor in cut-off mode with a size equal to the difference in size between the main bipolar pair, $Q_{1}$ and $Q_{2}$ [11]. This new transistor is $Q_{3}$ in Fig. 2. The sole purpose of this transistor is to ensure that CS leakage currents in both half circuits are matched, therein keeping collector currents equal even at high temperatures and thus compensating for the non-linearities arising from CS leakage. 
Note that Radoiaş et al. connected the emitter and base of $Q_{3}$ to VPTAT [11]. In contrast, in this work, we connect it to ground to avoid introducing non-linearities in VPTAT (and subsequently in $V R E F$ ) caused by the CB leakage of $Q_{3}$.

\subsection{Effects of collector-base, body-drain and source- body leakage current in bandgap circuits}

Excess current introduced in the bases of the bipolar pair, node B in Fig. 2, may adversely affect circuit operation, especially for low-power circuits [7]. Such an excess current arises at high temperatures due to $\mathrm{CB}, \mathrm{BD}$ or $\mathrm{SB}$ leakage introduced by reverse-biased p-n junctions in $Q_{1}$, $Q_{2}, M_{3}$ and $M_{\mathrm{S} 2}$, see Figs. 3 and 4 . In these figures, CB leakage occurs in $Q_{1}, Q_{2}$, and $Q_{3}$; BD leakage occurs in $M_{3}$, while SB leakage occurs in $M_{\mathrm{S} 2}$. Note that $M_{\mathrm{S} 2}$ is leaking current out of node $\mathrm{B}$, while the other leakages are leaking current into node B. However, summing all leakages from/to node $\mathrm{B}$ results in a net outflow of current from the node because the leaking junction area of the bipolar transistors are significantly bigger than that of $M_{\mathrm{S} 2}$.

Because $Q_{1}$ is biased at a higher current density than is $Q_{2}$ and has no series resistor, the majority of excess current injected in node $B$ will take the path through the $\mathrm{BE}$ junction of $Q_{1}$, be amplified by $Q_{1}$ and mirrored by the current mirror $M_{1}-M_{2}$. The gate voltage of $M_{3}$ will increase, whereby the current from $M_{3}$ supplied to node B will be reduced, counteracting the initially introduced current. However, if the loop gain of the $M_{1}-M_{2}-M_{3}$ feedback loop is low, non-negligible current imbalance between $Q_{1}$ and $Q_{2}$ will remain. Such an imbalance introduces non-linearities with respect to temperature in VREF and VPTAT. Because the CB junction of $Q_{3}$ is biased at a higher reverse voltage than are $Q_{1}$ and $Q_{2}$, the magnitude of its $\mathrm{CB}$ leakage current is slightly higher than that of $Q_{1}$ and $Q_{2}$. Thus, $M_{3}$ will subtract more current than are added in the form of $\mathrm{CB} / \mathrm{BD} / \mathrm{SB}$ leakage. At sufficiently high temperatures, $M_{3}$ will be turned completely off, whereas VREF and VPTAT will only be driven by leakage current, thus dropping to very low voltages.

Because $\mathrm{CB} / \mathrm{BD} / \mathrm{SB}$ leakage limits the high temperature range in which a bandgap sensor can operate, it is desirable to compensate for such leakage. Such compensation can be realised by adding artificial leakage sources in the form of transistors in cut-off with dimensions identical to that of the leaking transistors. This leakage can then be mirrored so that equal amounts of leakage is leaking into a node as is leaking out of it. Mizuno et al demonstrated such a compensation circuit in [6]. Because the direction of net leakage current in the Mizuno circuit (towards ground) is different from that in this work (towards supply), a modified circuit was presented in our previous work [7] and further extended in this work to compensate for one additional leaking transistor, $M_{\mathrm{S} 2}$. This circuit is presented in Fig. 5. Here, sources of artificial leakage are generated by $Q_{1}^{\prime}, Q_{1}^{\prime \prime}, Q_{2}^{\prime}, Q_{2}^{\prime \prime}, M_{3}^{\prime}$ and $M_{\mathrm{S} 2}^{\prime}$ operating in cut-off, while the current mirror consists of $M_{\mathrm{C} 1}, M_{\mathrm{C} 2}, M_{\mathrm{C} 3}, M_{\mathrm{C} 4}$ and $M_{\mathrm{C} 5}$. See [6] for details of how this circuit operates. A discussion of failure modes for the circuits of Figs. 4 and 5 is presented in Sect. 4.1.

\subsection{Matching of leakage currents}

The success of leakage current compensation techniques are highly dependent on the matching that can be achieved between the compensated and the compensating device. In their work, Mizuno et al have demonstrated matching of leakage currents with an error of 5\% [6]. This increased the temperature operating range by $20^{\circ} \mathrm{C}$ compared to using a simple current mirror as compensation circuit, by $40^{\circ} \mathrm{C}$ compared to using a diode directly and by $60^{\circ} \mathrm{C}$ compared to not using any leakage current compensation at all.

Because we use the same underlying compensation technique in this work for $\mathrm{CB} / \mathrm{BD} / \mathrm{SB}$ leakage current compensation, we expect a similar level of matching. Thus, we also expect the failure mechanisms involving by $\mathrm{CB} /$ $\mathrm{BD} / \mathrm{SB}$ leakage to be shifted up in temperature by approximately $60^{\circ} \mathrm{C}$.

\subsection{Power consumption of low-power, high- temperature bandgap sensors}

The power consumption, $P$, of a Brokaw bandgap reference with the two leakage current compensation circuits

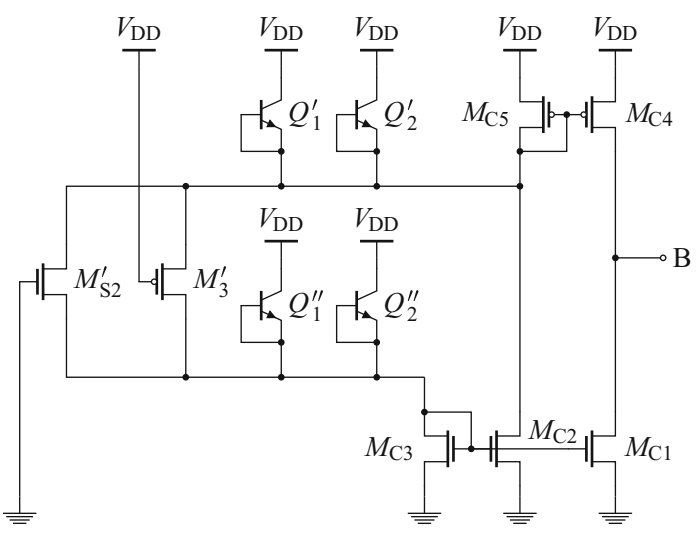

Fig. 5 Leakage current compensation circuit connected to the Brokaw bandgap circuit of Fig. 2. The prim/bis notation denotes devices with identical dimensions as similarly named devices from the Brokaw bandgap circuit depicted in Fig. 2. $M_{\mathrm{C} 1}, M_{\mathrm{C} 2}, M_{\mathrm{C} 3}, M_{\mathrm{C} 4}$ and $M_{\mathrm{C} 5}$, all have a width of $2 \mu \mathrm{m}$ and a length of $360 \mathrm{~nm}$. This circuit has been designed for a nominal supply voltage of $V_{\mathrm{DD}}=2.0 \mathrm{~V}$. The only currents flowing in the circuit are leakage current and compensation current (mirrored leakage current). The circuit is based on the work of Mizuno et al. [6] 
described earlier in this section consists of two temperature-dependent components; a linear part, due to bias current, and an exponential part, due to leakage current [7]. The power consumption can be written as

$$
P=c_{1} T+c_{2} \mathrm{e}^{b_{2} T},
$$

where $T$ is the temperature and $c_{1}, c_{2}$ and $b_{2}$ are linear and exponential temperature coefficients.

At low temperatures, the magnitudes of leakage currents are small and the linear part will dominate. At higher temperatures, the exponential part becomes significant and rapidly becomes the dominant term in the expression for the power consumption. Thus, there is a limit below which a reduction in bias current is not effective for reducing the power consumption at high temperatures where the exponential term is dominant.

\section{Experiments}

To assess the contribution of each compensation technique presented in Sect. 2, measurements were performed on three types of bandgap sensors:

- Type 0: With no leakage current compensation; that is, the circuit of Fig. 2 excluding $Q_{3}$.

- Type R: With the leakage current compensation circuit of Radioaş et al [11]; that is, the circuit of Fig. 2 including $Q_{3}$.

- Type RN: With the leakage current compensation circuit of Radioass et al [11] and the leakage current compensation circuit of Nilsson et al. [7]; that is, the circuit of Fig. 2 including $Q_{3}$ with the circuit of Fig. 5 connected to it (node B to node B).

Because of the high operating temperatures and relatively high output impedance of VREF and VPTAT, bootstrapped guard rings [1] were implemented on the printed circuit board (PCB) where the device under test was bonded, and polytetrafluoroethylene-insulated (PTFE) wires were used for connecting this board to the remainder of the measurement set-up. The guard rings were implemented because surface leakage currents in the PCB were observed at high temperatures. Driving a guard ring to the same potential as that of the trace which it surrounds minimises this leakage. For each measurement the temperature was required to remain within $\pm 1{ }^{\circ} \mathrm{C}$ (as measured using a Pt100 resistance thermometer) for $90 \mathrm{~s}$, after which 50 measurements were taken. Because of problems with tuning the oven temperature regulator at high temperatures, several devices were damaged by epoxy leaking from the PCB, thus limiting the number of samples that could be fully characterised.

\section{Measurement results}

A batch of $2.25 \mathrm{~mm}^{2}$ chips containing Type 0 , Type $\mathrm{R}$ and Type RN circuits were manufactured in a $0.18 \mu \mathrm{m}$ CMOS process with vertical npn BJTs. The dimensions used for the transistors are specified in Table 1. Temperature sweeps were performed on each type of circuit, and data for all the sweeps of VREF and VPTAT for the different types of circuits is presented in Fig. 6. Due to the difficulties encountered during the characterisations (as described in Sect. 3), we were only able to successfully complete temperature sweeps on 3 different Type 0 circuits, 5 different Type R circuits and 5 different Type RN circuits. Due to the limited sample size, we do not present standard deviations for the measured data.

Different properties of the different types of compensation circuits are analysed in the remainder of this section. Unless otherwise noted, the presented data is for a supply voltage $V_{\mathrm{DD}}$ of $2.0 \mathrm{~V}$.

\subsection{Failure modes}

From Fig. 6(a) it can be seen that the failure mode for Type 0 circuits follows the theory presented in Sect. 2.2. At moderately high temperatures both VREF and VPTAT increase, whereas at higher temperatures, they rapidly decrease to very low values.

For Type R circuits, it can be seen from Fig. 6(b) that for most circuits $V R E F$ and VPTAT become very low voltages at high temperatures. This is in agreement with the theory presented in Sect. 2.3. When $M_{3}$ is turned off, $V R E F$ is reduced to the point at which it triggers the startup circuit of Fig. 3. Because the start-up circuit is triggered at the threshold voltage of $M_{\mathrm{S} 1}, V R E F$ attains the value of said threshold voltage and because threshold voltage

Table 1 Dimensions of transistors from the circuits of Figs. 2, 3 and 5 for a $0.18 \mu \mathrm{m}$ design

\begin{tabular}{lcl}
\hline Device & & \\
\hline MOSFETs & Width (nm) & Length (nm) \\
\hline$M_{1}, M_{2}$ & 2000 & 360 \\
$M_{3}$ & 2000 & 720 \\
$M_{\mathrm{S} 1}, M_{\mathrm{S} 2}$ & 2000 & 360 \\
$M_{\mathrm{S} 3}, M_{\mathrm{S} 4}$ & 220 & 20000 \\
$M_{C 1}, M_{C 2}, M_{C 3}, M_{C 4}, M_{C 5}$ & 2000 & 3600 \\
\hline $\mathrm{BJTs}$ & Emitter area $\left(\mu \mathrm{m}^{2}\right)$ & \\
\hline$Q_{1}$ & $6.8(1$ device $)$ & \\
$Q_{2}$ & $40.8(6$ devices $)$ \\
$Q_{3}$ & $34.0(5$ devices $)$ \\
\hline
\end{tabular}




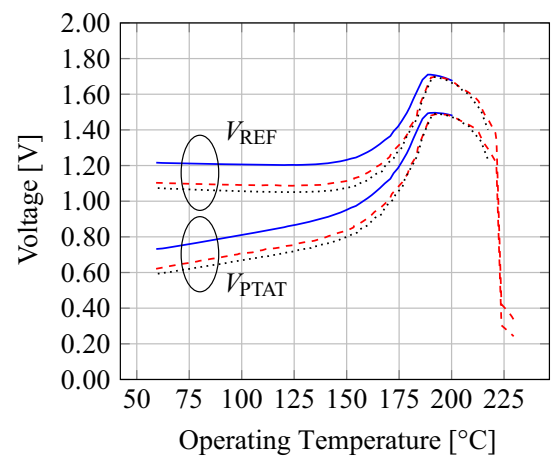

(a)

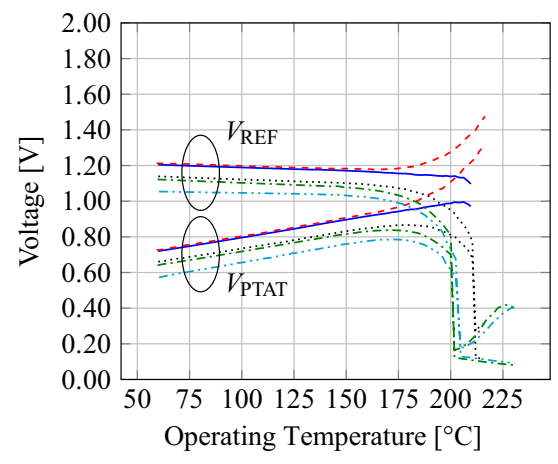

(b)

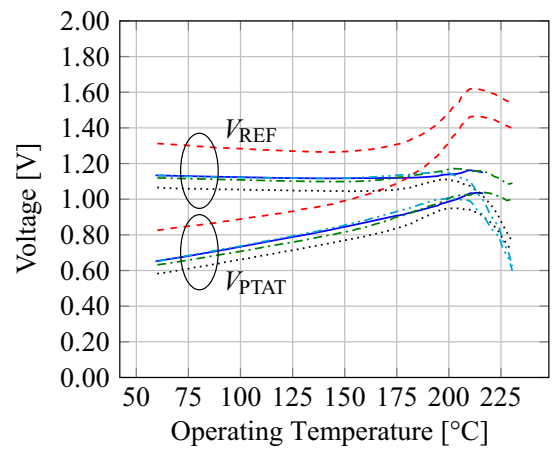

(c)
Fig. 6 Measurement results of $V R E F$ and $V P T A T$ as functions of temperature for different types of leakage current compensation circuits. Each plot contains data from multiple temperature sweeps

decreases with temperature [15], VREF also decreases with temperature.

As VREF decreases in Type $\mathrm{R}$ circuits, it can be seen from Fig. 6(b) that VPTAT starts to increase beyond $V R E F$. Because VPTAT is larger than $V R E F$ it can no longer be driven by $V R E F$. Thus, the current through $R_{2}$ must come from another place than node B. Looking at the circuit of Fig. 2, it can be seen that the current can only come from one or both of the bipolar transistors, $Q_{1}$ and $Q_{2}$. One feasible explanation for this behaviour is collector-emitter (CE) leakage current from pipe formation or surface inversion in the base [2]. Although surface inversion in bipolar devices is uncommon in modern processes, a reduced threshold voltage due to the unusually high operating temperature of the devices under test might trigger the phenomenon. Another source of CE leakage could be minority holes from the $\mathrm{CB}$ junction diffusing over to the BE junction instead of exiting through the base. This effect would be more prominent when the BE junction is reverse-biased, which it is at this operating point, because a reverse biased $\mathrm{BE}$ junction would decrease the effective base width. For the two temperature sweeps on Type $\mathrm{R}$ circuits where this effect could be observed, around $450 \mathrm{nA}$ was seen to run through $R_{2}$ at $225^{\circ} \mathrm{C}$.

One exception to the behaviour discussed above is the red, dashed line of Fig. 6(b). Here, VREF and VPTAT increase instead. One explanation for this deviation from the theory could be that for that particular circuit, a mismatch in the bipolar transistors occurred so that the left half-circuit of Fig. $2\left(Q_{1}\right.$ and $\left.Q_{3}\right)$ generates significantly less CS leakage current than the right half-circuit $\left(Q_{2}\right)$. The failure mode of this circuit then becomes similar to that of Type 0 circuits except that it occurs at higher temperatures because the CS leakage for each half-circuit is still better matched compared to Type 0 circuits.

From Fig. 6(c) it can be seen that for Type RN circuits similar non-linearities as for the Type R circuit are present, from different chips. Identical line styles in a plot indicate voltages from the same chip. a Type 0 . b Type R. c Type RN

although at higher temperatures compared to the Type $\mathrm{R}$ circuits. No rapid voltage reductions as seen in Type $\mathrm{R}$ circuits was seen in Type RN circuits at the examined temperatures. However, due to mismatches in compensated leakage currents resulting from process mismatches and/or the limitations of the compensation circuits used, it is likely that the Type RN circuits would fail in a manner similar to the other circuits, but that the failures occur at temperatures higher than the temperature range of the experiments performed in this work. As concluded in Sect. 2.4, if similar matching to that of [6] was achieved, this failure mode should occur for Type RN circuits at a temperature approximately $60^{\circ} \mathrm{C}$ higher than the temperature at which this happens for Type $\mathrm{R}$ circuits. However, the temperature sweeps performed in this work do not examine such high temperatures. Thus, this hypothesis has yet to be confirmed.

\subsection{Linearity, accuracy and temperature range}

For Type 0 circuits (Fig. 6(a)) significant non-linearities are introduced in $V R E F$ and VPTAT at temperatures as low as $150^{\circ} \mathrm{C}$. For Type $\mathrm{R}$ and Type $\mathrm{RN}$ compensations, it can be seen from Fig. 6(b), (c) that the same magnitude of non-linearities do not occur until above $175^{\circ} \mathrm{C}$.

Figure 7 shows a VPTAT/VREF ratio for different types of compensation circuit based on data of selected curves from Fig. 6 . The curves were not chosen to quantify performance, but rather to illustrate the characteristic behaviour of each of the different types of compensation circuit. The selected graphs are from sweeps from which the highest temperature ranges were obtained. For the Type 0 circuit, the curve is highly non-linear and experiences a rapid decrease at $220^{\circ} \mathrm{C}$, where both $V R E F$ and VPTAT are reduced rapidly. The Type $\mathrm{R}$ circuit achieves good linearity up to $210^{\circ} \mathrm{C}$, followed by a rapid increase in $V P T A T / V R E F$ where both VREF and VPTAT fall off 


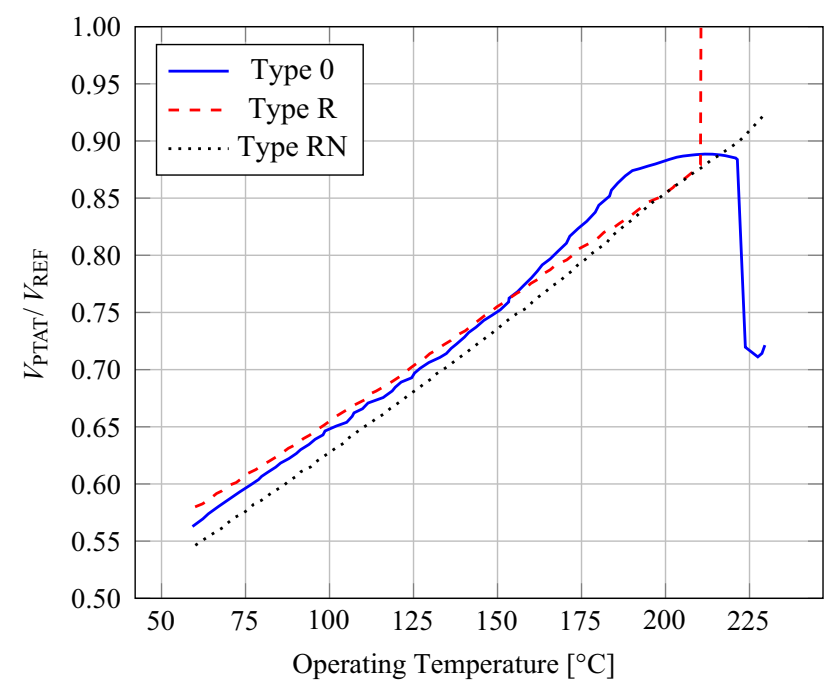

Fig. 7 Measurement results of $V P T A T / V R E F$ as functions of temperature for different types of leakage current compensation circuits for a Brokaw bandgap circuit. The Type 0 curve is derived from the red dashed curve from Fig. 6(a), and the Type R and RN curves are derived from the black dotted curves of Fig. 6(b), (c), respectively (Color figure online)

sharply. The Type RN circuit, on the other hand, presents good linearity over the entire $60-230^{\circ} \mathrm{C}$ temperature range.

The data of the VPTAT/VREF ratio was used to assess the linearity of the different types of compensation circuits and to relate the linearity to how it affects the temperature range for a given accuracy. Table 2 presents the best-case, median and worst-case ranges over which a least-squares fit to measured data in the $60-90^{\circ} \mathrm{C}$ temperature range produced a maximum deviation of 3,5 and $10^{\circ} \mathrm{C}$. We denote this deviation as the accuracy of the circuit. Examining the median upper temperature limits, it can be seen that the Type 0 circuit has the lowest upper temperature limit for all values of accuracy. For the 3 and $5^{\circ} \mathrm{C}$ accuracy requirements, the Type $\mathrm{R}$ circuit achieves the highest upper temperature limit, and the Type $\mathrm{RN}$ circuit achieves the highest upper temperature limit for an accuracy of $10^{\circ} \mathrm{C}$.

To further assess the accuracy of the circuits, histograms presenting the amount of chips which achieved a given accuracy at different temperatures are presented in Fig. 8. It can be seen from Fig. 8 (a) that at $195^{\circ} \mathrm{C}$, all of the 5 measured Type $\mathrm{R}$ circuits achieve an accuracy better than $7.5^{\circ} \mathrm{C}$. This is in contrast to the Type $\mathrm{RN}$ circuits for which the accuracies are generally worse and varies more. Fig. 8(b) shows that 4 of the 5 measured Type RN circuits achieve an accuracy between 2.5 and $10.0^{\circ} \mathrm{C}$ whereas 3 of those stay below $7.5^{\circ} \mathrm{C}$. The histograms are shown at $195^{\circ} \mathrm{C}$ because several of the Type $\mathrm{R}$ circuits experienced malfunction at around $200^{\circ} \mathrm{C}$. Looking at a higher temperature of $216^{\circ} \mathrm{C}$, it can be seen from Fig. 8(c) that the accuracy for Type RN devices is slightly reduced but still stays better than $7.5^{\circ} \mathrm{C}$ for 3 of the 5 tested chips. Data of Type $\mathrm{R}$ devices for $216^{\circ} \mathrm{C}$ is not presented because the Type $\mathrm{R}$ devices that were characterised up to that temperature experienced malfunction at $210^{\circ} \mathrm{C}$ or lower.

\subsection{Reference voltage stability}

Table 2 also shows the maximum $V R E F$ variation over the temperature range where the accuracy requirement is not exceeded. For the chips with median upper temperature limit, it can be seen that the Type RN circuit has a generally more stable $V R E F$ over temperature than has the Type $\mathrm{R}$ circuit.

The obtained results were compared to the simulations of [7]. For Type RN circuits with an accuracy of $3^{\circ} \mathrm{C}$ the median upper temperature limit is $168^{\circ} \mathrm{C}$ for which $V R E F$ varies by $16 \mathrm{mV}$. This result is in agreement with expected values based on simulation results from [7]. There, a Monte Carlo simulation showed a variation of $V R E F$ of $9.4 \mathrm{mV}$ over the 0 to $175^{\circ} \mathrm{C}$ temperature range, with a standard deviation of $6.8 \mathrm{mV}$.

As can be seen from Fig. 6(a), the VREF stability of the Type 0 circuit is generally low. However, we do not compare it to the other types of circuits because its upper temperature limit is significantly lower than that of the other circuits.

\subsection{Supply voltage requirements}

When repeating the above experiments for a supply voltage $V_{\mathrm{DD}}=2.5 \mathrm{~V}, V R E F$ and VPTAT were largely unaffected for most sweeps. However, for two out of thirteen measurements, a difference was seen at high temperatures. One possible explanation is that the hole mobility decreases with increasing temperature and a larger overdrive voltage over the $M_{1}-M_{2}$ mirror is required for a given current for the mirror to remain in saturation. In addition, leakage currents from the collectors of $Q_{1}$ and $Q_{2}$ must be resupplied by the current mirror, further increasing the required overdrive voltage. This phenomenon was seen for one of the Type 0 circuits, none of the Type $\mathrm{R}$ circuits and one of the Type RN circuits.

\subsection{Power consumption}

A plot of the mean power consumption based on four temperature sweeps of the Type RN circuit is shown in Fig. 9. In accordance with the theory presented in Sect. 2.5, the power consumption consists of a linear term and an exponential term. The exponential coefficient $b_{2}$ was obtained from the exponential part of the plotted data and found to be $0.064 \mathrm{C}^{-1}$. This supports the theory that the 
Table 2 Upper temperature limits for different types of leakage current compensation circuits for different values of accuracy (Color table online)

\begin{tabular}{|c|c|c|c|}
\hline Accuracy $\left({ }^{\circ} \mathrm{C}\right)$ & \multicolumn{2}{|c|}{ Upper Temperature Limit } & $\left({ }^{\circ} \mathrm{C}\right)$ \\
\hline & Type 0 & Type R & Type RN \\
\hline 10 & $171 / 162 / 161$ & $216 / 210 / 200$ & $228 / 216 / 181$ \\
\hline 5 & $156 / 155 / 153$ & $208 / 200 / 184$ & $202 / 182 / 158$ \\
\hline \multirow[t]{3}{*}{3} & $150 / 147 / 144$ & $200 / 170 / 162$ & $182 / 168 / 147$ \\
\hline & \multicolumn{3}{|c|}{ Maximum $V_{\mathrm{REF}}$ Variation $(\mathrm{mV})$} \\
\hline & Type 0 & Type $\mathrm{R}$ & Type RN \\
\hline 10 & $140 / 74 / 65$ & $258 / 303 / 283$ & $257 / 46 / 53$ \\
\hline 5 & $42 / 30 / 28$ & $270 / 283 / 36$ & $22 / 22 / 48$ \\
\hline 3 & $24 / 22 / 16$ & $283 / 36 / 32$ & $17 / 16 / 48$ \\
\hline
\end{tabular}

For each type of compensation and for a certain accuracy, the best, median and worst chips in terms of highest upper limits are shown in the following format: " $<$ best-case $>/<$ median $>/<$ worst-case $>$ ". The maximum $V R E F$ variation over the range where the accuracy is not exceeded is also shown. An identical position or colour for a given accuracy indicates data from the same chip

exponential term is due to reverse leakage current, which approximately doubles for every $10^{\circ} \mathrm{C}$ increase in temperature [13].

At $60^{\circ} \mathrm{C}$, the power consumption of the different chips ranged from 230 to $320 \mathrm{nW}$ (mean $260 \mathrm{nW}$ ), which is a relative variation of $32 \%$. At $200^{\circ} \mathrm{C}$, the power consumption ranged from 1.5 to $2.8 \mu \mathrm{W}$ (mean $2.1 \mu \mathrm{W}$ ), reaching the higher relative variation of $60 \%$, and at $230^{\circ} \mathrm{C}$, the power consumption ranged from 9.5 to $19 \mu \mathrm{W}$ (mean $14 \mu \mathrm{W})$, reaching a relative variation of $67 \%$. This variation of power consumption between different regions of operation suggests that the power consumption generated by leakage current is more sensitive to process variation than is the power consumption generated by bias current.

\section{Discussion}

From the results presented in Sect. 4.2, it can be seen that two aspects play an important role when determining which compensation type to use when designing a lowpower, high-temperature bandgap temperature sensor: how important it is to generate a stable reference voltage and how high of a measurement accuracy that is required.

\subsection{Stable reference voltage}

An advantage of using a bandgap circuit as a temperature sensor is that one of the generated output voltages is a relatively temperature-invariant reference voltage that can be used by other parts of the circuit that is not directly taking ratiometric temperature measurements. For example, an ADC may be needed to obtain digital samples of the measured voltages, or a voltage regulator in a mixed-signal design may need a stable voltage reference to operate properly. Thus, depending on the system specifications, because a Type RN circuit outperforms a Type $\mathrm{R}$ circuit in terms of $V R E F$ stability, it may be preferable to use a Type RN circuit even though its temperature range is generally lower for an accuracy of $5^{\circ} \mathrm{C}$ or better.

\subsection{Accuracy and temperature range}

If an accuracy better than $5^{\circ} \mathrm{C}$ is required, it has been demonstrated that the Type $\mathrm{R}$ circuit provides the highest operating temperature range. However, if the accuracy requirement is relaxed to $10^{\circ} \mathrm{C}$, which may be acceptable for a condition monitoring system, the Type RN circuit achieves the higher range.

Although most samples of the Type R circuit outperforms the Type $\mathrm{RN}$ circuit in terms of temperature range at better accuracies than $5^{\circ} \mathrm{C}$, some Type $\mathrm{R}$ chips were shown to suffer from rapid reductions in output voltage at a specific temperature where both VREF and VPTAT fall off sharply. If $V R E F$ becomes small, voltage regulators and subsequently digital subsystems requiring a regulated voltage in order to operate will shut down, reducing the possibilities for the system to signal that there is a problem. This behaviour was not seen for any of the Type RN chips in the temperature range where the experiments were performed, that is, from 60 to $230^{\circ} \mathrm{C}$. Thus, it would appear that Type RN circuits could operate at a higher temperature range, although with lower accuracy, while keeping a digital subsystem operational.

Another aspect of the fact that no such malfunction was seen for Type RN circuits is that the accuracy can be significantly improved if the system is calibrated digitally, and the difference in accuracy between a Type R and Type RN compensation circuit can be minimised, and thus, the operating range for a certain amount of accuracy can be extended. For circuits suffering from this type of malfunction, such a calibration will not improve the accuracy after the rapid voltage reduction point because too severe non-linearities are introduced there.

\subsection{Comparison to other work}

Table 3 presents a comparison of the sensor in this work in terms of different requirements on accuracy with state-ofthe-art low-power, high-temperature sensors. The sensors in this work have no integrated ADC, and thus no power consumption value for those positions. Future work 


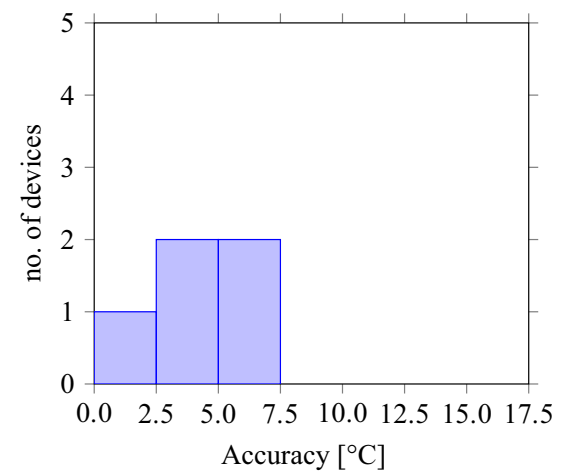

(a)

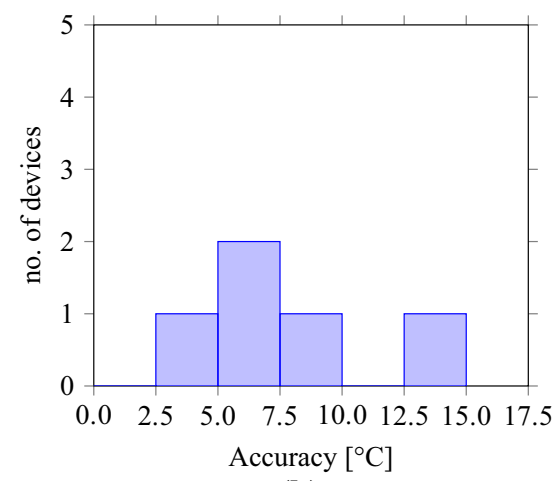

(b)

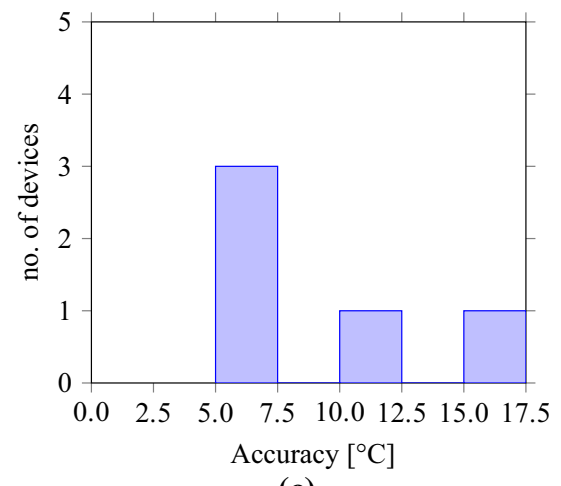

(c)
Fig. 8 Histograms of the accuracy for Type R and Type RN circuits for $195^{\circ} \mathrm{C}$ and $216^{\circ} \mathrm{C}$. Data for each plot is derived from 5 temperature sweeps of different chips for both Type $\mathrm{R}$ and Type RN circuits. Data for Type R circuits at $216^{\circ} \mathrm{C}$ has been omitted because

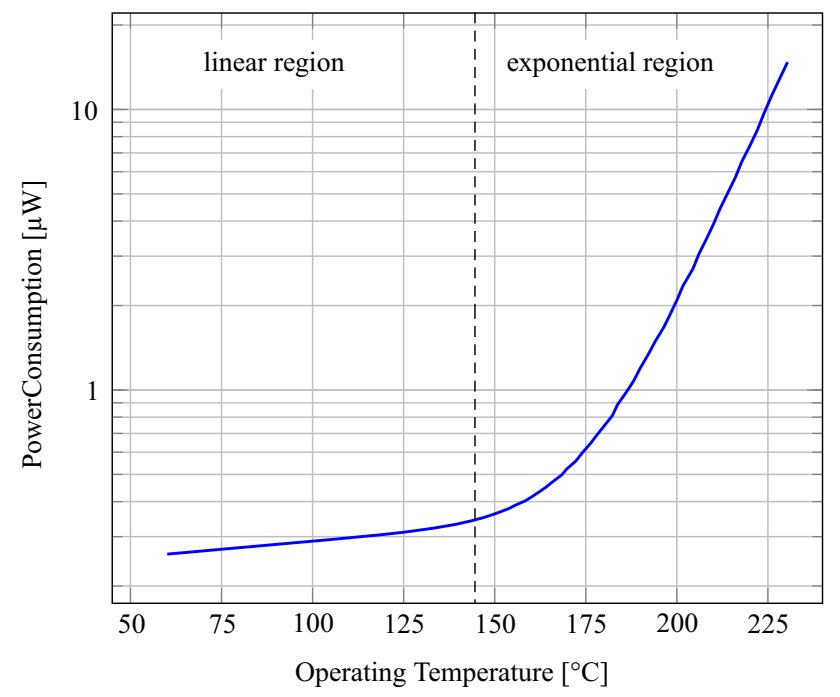

Fig. 9 Mean power consumption of the Type RN circuit based on measured data from four temperature sweeps all Type $\mathrm{R}$ devices characterised at said temperature experienced malfunction at $210^{\circ} \mathrm{C}$ or lower, as can be seen from Fig. 6(b). a Type $\mathrm{R}, 195^{\circ} \mathrm{C}$. b Type RN, $195^{\circ} \mathrm{C}$. c Type RN, $216^{\circ} \mathrm{C}$

includes investigating how to combine a power-efficient ADC with the circuits presented in this work.

The sensors from this work show a higher or equal upper temperature limit compared to all other sensors with a bandgap as the sensing element. However, this is achieved at the expense of accuracy. For the case of a sensor in this work with an accuracy of $5^{\circ} \mathrm{C}$, the same upper temperature limit is obtained as in [16], and the power consumption is smaller by a factor of 17. However, [16] achieves an accuracy as high as $0.4^{\circ} \mathrm{C}$.

The only non-bandgap sensor in this comparison, [12], achieves an upper temperature limit as high as $250^{\circ} \mathrm{C}$. This is significantly higher than the sensor of this work even if an accuracy of only $10^{\circ} \mathrm{C}$ is accepted. However, this is achieved with the power consumption increased by a factor of 18 compared to the sensors of this work.

In contrast to the other sensors in this comparison, the sensors from this work were not manufactured in an

Table 3 Comparison of the performance of different temperature sensors

\begin{tabular}{lllllll}
\hline Parameter & This work $a$ & This work $b$ & This work $c$ & {$[9]$} & {$[16]$} & {$[12]$} \\
\hline Sensing element & Bandgap & Bandgap & Bandgap & Bandgap & Bandgap & PIN diode \\
SOI & No & No & No & Yes & Yes & Yes \\
Calibration points & 2 & 2 & 2 & 1 & 1 & 2 \\
Process technology node $(\mu \mathrm{m})$ & 0.18 & 0.18 & 0.18 & 1.0 & 0.16 & Not available \\
Power consumption (including ADC) $(\mu \mathrm{W})$ & Not available & Not available & Not available & 90 & 40 & 150 \\
Power consumption $($ excluding ADC) $(\mu \mathrm{W})$ & 5.7 & 2.1 & 0.53 & 45 & 22 & 12 \\
Accuracy $\left({ }^{\circ} \mathrm{C}\right)$ & 10 & 5 & 3 & 1.6 & 0.4 & 3 \\
Temperature range $\left({ }^{\circ} \mathrm{C}\right)$ & $0-216$ & $60-200$ & $60-170$ & $40-170$ & $-55-200$ & $25-250$ \\
\hline
\end{tabular}

This work is included three times to demonstrate how the performance parameters change with changing requirements on accuracy. Power consumption is taken as the highest value over the entire temperature range. This table is based on the comparison table found in [9]

a The Type RN circuit corresponding to the measurements of the blue solid line of Fig. 6(c).

$\mathrm{b}$ The Type R circuit corresponding to the measurements of the green dashed-dotted line of Fig. 6(b).

c The Type R circuit corresponding to the measurements of the red dashed line of Fig. 6(b) 
silicon on insulator (SOI) process to achieve a high upper temperature limit. Because leakage currents are generally smaller in SOI processes, it is possible that the temperature range could be further extended and/or the power consumption could be reduced using such a process.

\section{Conclusion}

Experiments show that Type $\mathrm{R}$ and Type RN circuits extend the temperature range compared to the Type 0 circuit by $20-50^{\circ} \mathrm{C}$. For an accuracy of $10^{\circ} \mathrm{C}$ or more, the Type RN circuit achieves a higher upper temperature limit compared to the Type $\mathrm{R}$ circuit, and for an accuracy of $5{ }^{\circ} \mathrm{C}$ or less, the Type $\mathrm{R}$ circuit achieves the higher limit. Some of the examined Type $\mathrm{R}$ circuits were seen to experience a total circuit malfunction above a certain temperature where no temperature readings can be conducted. This phenomenon was not seen for any of the Type $\mathrm{RN}$ circuits over the entire temperature range from 60 to $230^{\circ} \mathrm{C}$.

At high temperatures, the power consumption of the temperature sensors was found to be highly dependent on leakage current. It was seen to increase exponentially with increasing temperature, setting a limit at which it is no longer effective to reduce the power consumption by reducing bias current.

The sensors presented in this work are suitable for use in the field of condition monitoring of power semiconductors. Their high upper temperature limit and low power consumption make direct-contact sensors suitable for wireless single-chip designs. A drawback to using these types of sensors is their low accuracy; however, the tradeoff between high temperature operation and high accuracy may be acceptable depending on the system requirements. Furthermore, the accuracy may be improved by calibrating the sensors digitally.

Acknowledgements The work presented in this paper was Funded by Svenska Kraftnät.

Open Access This article is distributed under the terms of the Creative Commons Attribution 4.0 International License (http://creative commons.org/licenses/by/4.0/), which permits unrestricted use, distribution, and reproduction in any medium, provided you give appropriate credit to the original author(s) and the source, provide a link to the Creative Commons license, and indicate if changes were made.

\section{References}

1. Ardizzoni, J. (2005). A practical guide to high-speed printedcircuit-board layout. Analog Dialogue 39. http://www.analog. com/en/analog-dialogue/articles/high-speed-printed-circuit-boardlayout.html.
2. Barson, F. (1976). Emitter-collector shorts in bipolar devices. IEEE Journal of Solid-State Circuits, 11(4), 505-510. doi:10. 1109/JSSC.1976.1050767.

3. Brokaw, A. (1974). A simple three-terminal IC bandgap reference. IEEE Journal of Solid-State Circuits, 9(6), 388-393. doi:10.1109/JSSC.1974.1050532.

4. Held, M., Jacob, P., Nicoletti, G., Scacco, P., \& Poech, M. H. (1997). Fast power cycling test of IGBT modules in traction application. In Proceedings of 1997 international conference on power electronics and drive systems, 1997, vol. 1, (pp. 425-430). doi:10.1109/PEDS.1997.618742.

5. Meijer, G. C. (1986). Thermal sensors based on transistors. Sensors and Actuators, 10(1-2), 103-125. doi:10.1016/02506874(86)80037-3. http://www.sciencedirect.com/science/article/ pii/0250687486800373.

6. Mizuno, K., Ohta, N., Kitagawa, F., \& Nagase, H. (1998). Analog CMOS integrated circuits for high-temperature operation with leakage current compensation. In HITEC 1998 fourth international high temperature electronics conference, 1998, IEEE, (pp. 41-44).

7. Nilsson, J., Borg, J., \& Johansson, J. (2015). Leakage current compensation for a $450 \mathrm{nW}$, high-temperature, bandgap temperature sensor. In 22nd international conference on mixed design of integrated circuits systems (MIXDES), 2015 (pp. 343-347). doi:10.1109/MIXDES.2015.7208540.

8. Nilsson, J., Borg, J., \& Johansson, J. (2015). Single chip wireless condition monitoring of power semiconductor modules. In NORCHIP international symposium on system-on-chip (SoC), 2015 nordic circuits and systems conference (NORCAS), (pp. 1-4). doi:10.1109/NORCHIP.2015.7364407.

9. Pathrose, J., Liu, C., Chai, K., \& Xu, Y. P. (2015). A time-domain band-gap temperature sensor in SOI CMOS for high-temperature applications. IEEE Transactions on Circuits and Systems II: Express Briefs, 62(5), 436-440. doi:10.1109/TCSII.2014. 2386231.

10. Radoiaş, L., Zegheru, C., \& Brezeanu, G. (2012). Substrate leakage current influence on bandgap voltage references in automotive applications. In 2012 International Semiconductor Conference (CAS), vol. 2, (pp. 389-392). doi:10.1109/SMICND. 2012.6400752.

11. Radoiaş, L., Zegheru, C., \& Brezeanu, G. (2013). Sustrate leakage compenstaion technique for low quiescent current bandgap voltage references. UPB Scientific Bulletin, Series C: Electrical Engineering, 75(4), 69-78.

12. Rue, B., \& Flandre, D. (2007). A SOI CMOS smart high-temperature sensor. In 2007 IEEE international SOI conference, (pp. 111-112). doi: 10.1109/SOI.2007.4357877.

13. Sedra, A. S., \& Smith, K. C. (2010). Microelectronic circuits (6th ed.). Oxford: Oxford University Press.

14. Shockley, W. (1949). The theory of p-n junctions in semiconductors and p-n junction transistors. The Bell System Technical Journal, 28(3), 435-489. doi:10.1002/j.1538-7305.1949. tb03645.x.

15. Shoucair, F. (1986). Design consideration in high temperature analog CMOS integrated circuits. IEEE Transactions on Components, Hybrids, and Manufacturing Technology, 9(3), 242-251. doi:10.1109/TCHMT.1986.1136646.

16. Souri, K., Souri, K., Makinwa, K.: A $40 \mu \mathrm{W}$ CMOS temperature sensor with an inaccuracy of $\pm 0.4^{\circ} \mathrm{C}(3 \sigma)$ from $-55^{\circ} \mathrm{C}$ to $200^{\circ}$ C . In Proceedings of the ESSCIRC (ESSCIRC), 2013, (pp. 221-224) (2013). doi:10.1109/ESSCIRC.2013.6649112.

17. Yang, S., Xiang, D., Bryant, A., Mawby, P., Ran, L., \& Tavner, P. (2010). Condition monitoring for device reliability in power electronic converters: A review. IEEE Transactions on Power Electronics, 25(11), 2734-2752. doi:10.1109/TPEL.2010. 2049377. 


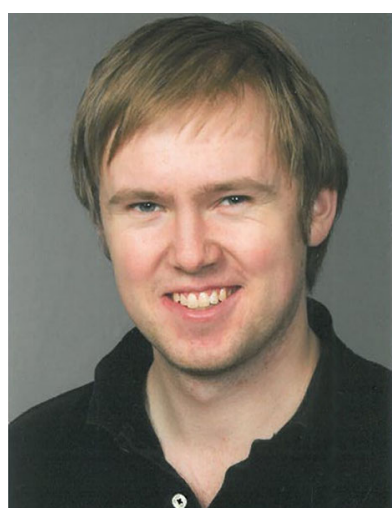

Joakim Nilsson was born in 1989. He received his M.Sc. degree in engineering physics and electrical engineering from the Luleå University of Technology, Luleå, Sweden in 2013, from where he has been pursuing his Ph.D. studies in industrial electronics since 2014. His research interests include integrated circuit design for lowpower sensor systems and semiconductor physics.

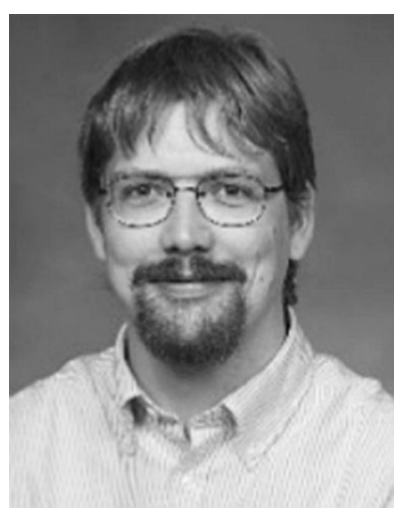

Johan Borg was born in 1978. He received his M.Sc. degree in applied physics and electrical engineering from Linköping University, Linköping, Sweden in 2003, and his Ph.D. degree from the Lulea University of Technology, Luleå, Sweden in 2010. He continued at Luleå University of Technology as an Associate Senior Lecturer, and later Senior Lecturer, until 2016 when he joined the High Energy Physics group of the Department of Physics at Imperial College London, United Kingdom, where he is currently an
Electronics Research Engineer. His research interests include integrated circuit design for sensor systems.

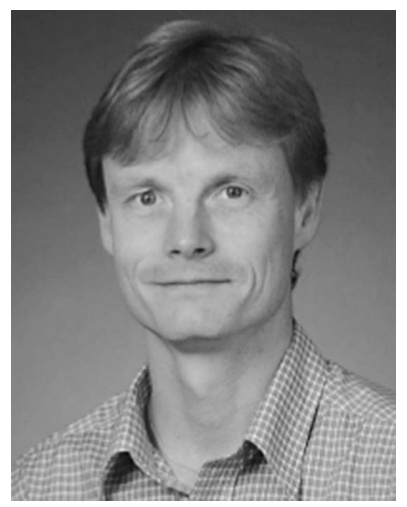

Jonny Johansson was born in 1968. He received his M.Sc. degree in electrical engineering from the Chalmers University of Technology, Gothenburg, Sweden in 1992 and his Ph.D. degree from the Luleå University of Technology, Luleå, Sweden in 2004. Between 1992 and 1999, he worked for Asea Brown Boveri, Ludvika, Sweden, where he was involved in the development of high-voltage measurement systems. In 1999, he joined EISLAB at the Luleå University of Technology, where he is currently an associate professor. His research interests include low-power mixed-mode designs for sensor systems. 